binding to the replisome clamp to mediate DNA loss.

To translate the bacterial data to human cancer, the team identified 284 human proteins that are homologous to the bacterial DDPs and found that their expression levels in cancer correlated with mutation load and poor clinical prognosis. These relationships held even when known cancer drivers were excluded, indicating that the set of candidate human DDPs contained currently underappreciated cancer-related proteins. It remains uncertain how many genuine human DDPs exist: of the candidate human DDPs tested, approximately half validated in DNA damage assays in human cells, but there may be additional human DDPs given the many human genes without bacterial homologues.

Regarding specific functional connections between bacterial and human DDPs, the DNA damage induced by the human potassium channel subunits KCNAB1 and KCNAB2 was connected to ROS elevation (similar to the bacterial $\mathrm{H}^{+}$symporters), which provides a rationale for how membrane proteins

present. Interestingly, low-scoring cryptic splice sites were often used in a tissue-specific manner, which suggests that tissue-specific features (such as chromatin state or complement of RNA-binding proteins) might drive the splice site choice when normal and novel sites are otherwise equally favourable.

The authors next sought to determine whether SpliceAl might improve the diagnostic yield of rare genetic disorders, which is currently only $25-30 \%$ with whole-exome sequencing. The neural network was used to predict the effects of de novo mutations in 4,293 individuals with intellectual disability from the Deciphering Developmental Disorders (DDD) cohort and 3,953 individuals with autism spectrum disorders (ASDs) from the Simons Simplex Collection and the Autism Sequencing Consortium. Splice-disrupting de novo mutations were significantly enriched in intellectual disability and ASD test groups compared with controls. The authors estimate that cryptic splice variants account for $9 \%$ of pathogenic mutations in intellectual can affect DNA fidelity from a distance. Furthermore, similar to bacterial DNA polymerase IV, human DNA methyltransferase 1 (DNMT1) caused DNA damage even without catalytic activity and bound to the replisome clamp, indicating that oncogenic roles of DNMT1 might extend beyond silencing of tumour suppressor genes through promoter DNA methylation.

This study highlights how a bacterial system can provide relevant functional information for human cancer, despite their evolutionary distance. Notable avenues for future investigation include assessing whether DDP levels in cancer provide distinct prognostic and therapeutic information relative to sequencing-based cancer mutation signatures and whether human oncogenic transcription factors have additional replication fork effects beyond known roles in gene dysregulation.

Darren J. Burgess

ORIGINAL ARTICLE Xia, J., Chiu, L.-Y. et al. Bacteria-to-human protein networks reveal origins of endogenous DNA damage. Cell 176, 127-143 (2019)

disability and $11 \%$ in ASD. Importantly, RNA-seq analysis of lymphoblastoid cell lines derived from 28 individuals with ASD from the Simons Simplex Collection validated the predicted cryptic splicing pattern in 21 of those individuals, which suggests that most predictions are likely to be functionally relevant. Although the seven non-validated mutations could be false-positive predictions, it is also possible that they failed to validate owing to tissue-specific splicing at the cryptic splice site.

The authors suggest that cryptic splice mutations might explain a similar percentage (9-11\%) of cases for other rare genetic disorders. With this in mind, they have made available prediction scores for every possible nucleotide substitution genome-wide. This resource should facilitate the interpretation and understanding of cryptic splice variation in genetic disease.

Dorothy Clyde

ORIGINAL ARTICLE Jaganathan, K. et al. Predicting splicing from primary sequence with deep learning. Cell https://doi.org/10.1016/ j.cell.2018.12.015 (2019)

\section{CLINICAL GENETICS}

\section{Baby sequencing steps}

The accurate diagnosis of a genetic condition at birth can benefit a child by giving access to tailored surveillance, treatment and management as well as information and support, such as adequate genetic counselling, for the individual and their families. The BabySeq project, a pilot randomized clinical trial exploring the value of routine genomic sequencing of neonates compared with standard newborn screening, now reports initial results in the American Journal of Human Genetics.

Although genomic sequencing can potentially shorten the diagnostic odyssey for patients with rare paediatric diseases, the analysis and interpretation of identified variants remains highly challenging, which has meant that the use of whole-exome sequencing (WES) and whole-genome sequencing as a diagnostic tool at birth remains far from routine. The BabySeq study enrolled 316 participants, including 251 healthy neonates and 65 neonates who had been admitted to newborn intensive care units (NICUs). A total of 157 neonates were randomly assigned to standard newborn screening, whereas the remaining 159 (127 healthy babies and 32 in NICUs) underwent WES in addition to standard newborn screening tests.

Only results for genes strongly associated with childhood-onset conditions were shared with parents. Of the sequenced study participants, $15(9.4 \%)-10$ healthy neonates and 5 admitted to the NICU - were found to harbour genetic variants that increase the risk of a childhood-onset disease or condition for which early treatment would be beneficial, such as cardiomyopathy, congenital adrenal hyperplasia or hearing loss. The results could not have been predicted on the basis of clinical or family history of these neonates.

A total of $88 \%$ of sequenced neonates had carrier status for one or more rare genetic variants known to be associated with recessive diseases, that is, they were not at risk of the disease themselves but could pass disease-causing genetic variants on to their offspring. Carrier status for pharmacogenomic variants, that is, variants that influence the response to drugs used in the paediatric patient population, were identified in $5 \%$ of study participants undergoing WES. About half of the parents agreed to receive WES results about actionable adult-onset conditions, such as breast or colon cancer, which were found in three babies (3.5\%), prompting referral for counselling and potential surveillance for the parent who had passed on these variants.

The BabySeq Project will continue to follow up the sequenced study participants to determine the economic, medical and behavioural impact of WES on the families over time.

Linda Koch

ORIGINAL ARTICLE Ceyhan-Birsoy, O. et al. Interpretation of genomic sequencing results in healthy and ill newborns: results from the BabySeq Project. Am.J. Hum. Genet. 104, 76-93 (2019)

FURTHER READING Wright, C. F. et al. Paediatric genomics: diagnosing rare disease in children. Nat. Rev. Genet. 19, 253-268 (2018) 